\title{
Coronavirus jolts labs to warp speed
}

To thwart SARS-CoV-2, labs hurry to hone assays, swap findings and share resources.

\author{
Vivien Marx
}

"S afety is my biggest concern. STAY HOME," writes microbiologist Julie Pfeiffer from University of Texas Southwestern Medical Center to her team. She shuttered the lab in mid-March. If members of her lab go out for essentials, she recommends they constantly disinfect their hands, avoid touching their face and steer clear of crowds. Hypervigilance is called for should essential tasks bring them on campus. They need to, for example, wipe door handles as they go. "As virologists, it's critical that we help 'flatten the curve' by doing our best to not become infected and not spread SARS-CoV-2 to others. You know aseptic technique; use it in your daily activities," writes Pfeiffer.

COVID-19 has gripped the world as a readily transmissible respiratory illness that can take a severe course. The staggering worldwide infection rates and deaths are tracked in a dashboard from the Johns Hopkins University Center for Systems Science and Engineering. A variety of hurdles always threaten to slow things, but labs find ways to keep accelerating their work and their exchanges about it. Scientists have collaboratively discovered, for example, what structural traits could explain how easily cells can be infected by severe acute respiratory syndrome coronavirus 2 , or SARS-CoV-2, as it was named by the International Committee on Taxonomy of Viruses. Labs have worked out better ways to culture the virus, assay it reliably and at scale, and perform genetic and phylogenetic analyses. On our blog are more COVID-19 news stories.

"It's exhilarating to be working at this pace," says microbiologist Nicholas Loman from the University of Birmingham in the United Kingdom. He and his team have set up to sequence samples from all the COVID-19 cases in Birmingham and the surrounding areas in real time, as well as support over 50 groups to achieve the same in over 20 countries. Most recently the first genomes from Brazil, New Zealand, Scotland, Wales and the Democratic Republic of the Congo have arrived. "It is great, but there is an awful lot of WhatsApp and Slack messages to deal with," he says. Loman likes the discussions on Virological. org, which is devoted to analysis and interpretation of virus molecular evolution

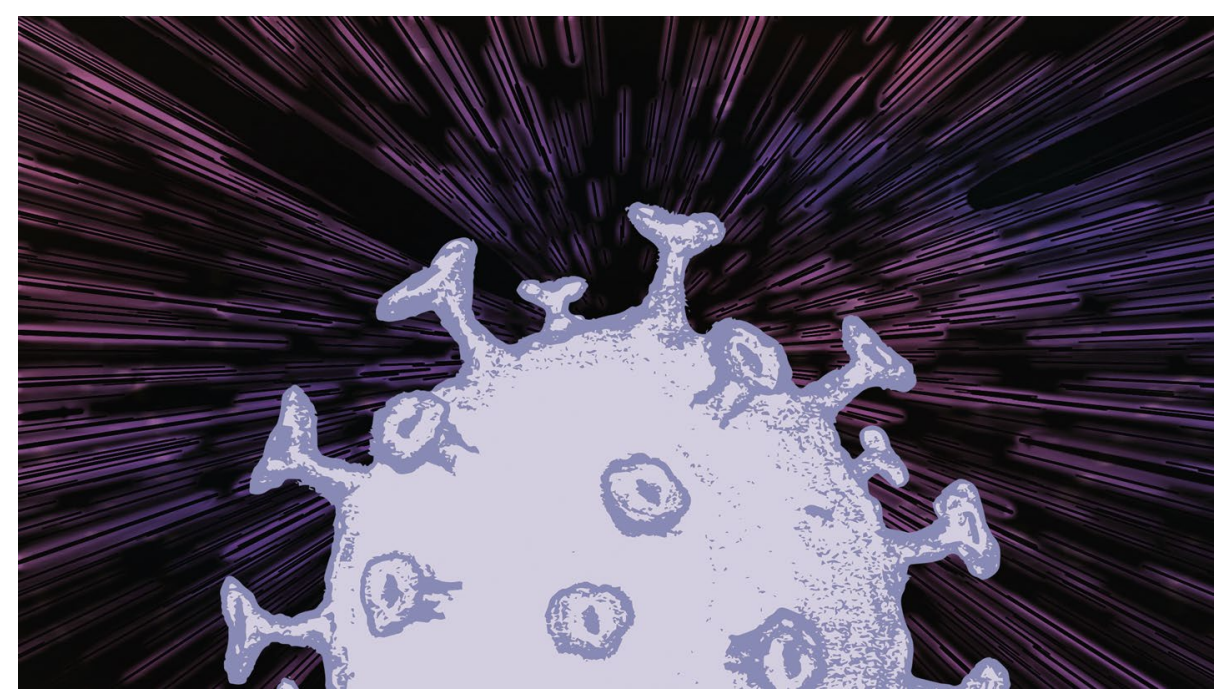

The new coronavirus SARS-CoV-2 grips the world. Researchers collaborate intensively, swapping data, sharing insight and resources to battle this virus and the illness it causes, COVID-19. Credit: M. Spence, Springer Nature/E. Muzhevsky Science photo library/Getty

and epidemiology. Data from, for example, the Global Initiative on Sharing All Influenza Data (GISAID) feed into Nextstrain, which is devoted to the exchange of genome data about pathogens and which offers a suite of software tools. Nextstrain has a dedicated tab for genetic epidemiological analysis of the novel coronavirus, with interactive

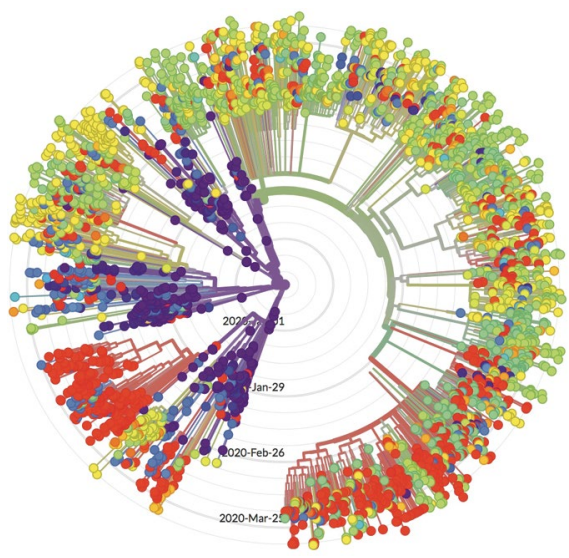

Labs rapidly share data to study the genomic epidemiology of the novel coronavirus. Here, a radial phylogenetic tree of over 3,900 genomes of the virus. Credit: Nextstrain.org data visualizations. Coronavirus genomes are uploaded as soon available. As Nature Methods went to press, the site had over 3,900 genomes.

Some labs have struggled to culture SARS-CoV-2. Alyssa Pyke, Ian Mackay and colleagues at Queensland Health in Australia developed an approach and quickly shared it; they note that extensive cell culture experience and PC3 biological containment conditions are needed to use it. Mount Sinai Icahn School of Medicine microbiologist Benjamin tenOever and team, including colleagues Wen-Chun Liu and Randy Albrecht, shared their plaquing assay for SARS-CoV-2, which is a way to quantify virus particles in a sample. "Plaquing some viruses can be tricky," says tenOever. Other assays work, but plaque-forming units per milliliter "is the gold standard."

\section{What's that spike?}

A genomic analysis of the virus at Fudan University in Shanghai; several Wuhan institutions, including the Wuhan Center for Disease Control; the China Center for Disease Control and Prevention in Beijing; and the University of Sydney was submitted 
in early January and published in early February ${ }^{1}$. The researchers sequenced the 29,903 nucleotides of the "new coronavirus" isolated from bronchoalveolar lavage fluid from a 41-year old patient experiencing severe respiratory disease who had been admitted to the Central Hospital of Wuhan in late December. They also presented a phylogenetic analysis. The team performed metagenomic RNA sequencing with the Illumina Mini-Seq system, generating 56 million reads, and assembled nearly 400,000 contiguous segments with the software tool Megahit. By the time the paper was published, the genome sequence in GenBank had been posted and updated multiple times. To explore infectiousness, the scientists used the algorithm MUSCLE to compare amino acid sequences from the receptor-binding domain (RBD) of this virus's spike protein with those of different SARS coronaviruses (CoVs) and SARS-like CoVs. The similarities between the RBD-related amino acid sequences and predicted protein structures of the novel coronavirus and SARS-CoV indicate that the novel virus can invade a cell by using the human angiotensin-converting enzyme 2 (ACE2) receptor as a kind of doorknob. Doing so, the authors note, "could potentially facilitate human-to-human transmission."

A number of structural biology labs have pounced on the viral spike. Insight builds on previous work ${ }^{2}$ from 2005 on the structure of the SARS coronavirus spike RBD complexed with ACE2. Some groups, such as David Veesler and colleagues at the University of Washington, with colleagues at Fred Hutchinson Cancer Research Center and Institut Pasteur ${ }^{3}$, used cryoelectron microscopy (cryo-EM). Other cryo-EM work ${ }^{4}$ comes from the lab of Jason McLellan at the University of Texas (UT) at Austin with colleagues at the National Institutes of Health's National Institute of Allergy and Infectious Diseases.

"This whole family of viruses makes use of these spikes, and each coronavirus has its own unique spike protein," says Daniel Wrapp, a member of the McLellan lab at UT Austin and co-first author of the Science paper ${ }^{4}$ on the cryo-EM structure of the viral spike. The more closely related two viruses are, such as SARS-CoV and SARS-CoV-2, the more similar their spikes. The team points out the spike is a key target for vaccines, therapeutic antibodies and diagnostics. Structural data will also help with evaluating ongoing spike mutations as the virus undergoes genetic drift. The University of Washington team notes the SARS-CoV-2 spike glycoprotein has a furin cleavage site between protein subunits, an aspect that makes this virus unlike SARS-CoV and other SARS-related CoVs. They also show ACE2 could mediate

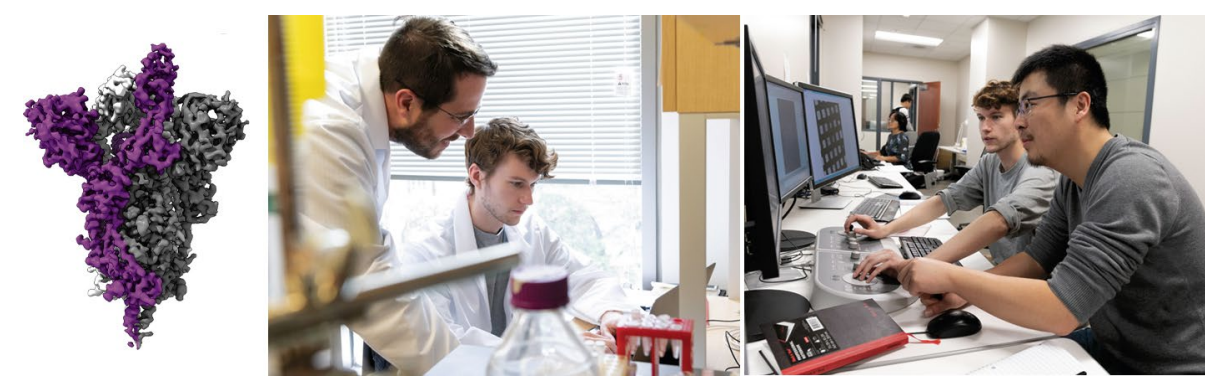

Cryoelectron microscopy is revealing how SARS-CoV-2 infects cells. From left: the viral spike protein; Jason McLellan (left) with graduate student Daniel Wrapp; Wrapp looking at cryo-EM data with colleague Nianshuang Wang (right). Credit: UT Austin

SARS-CoV-2 entry into cells. As Wrapp explains, "we were initially a little surprised to see the enhanced affinity between this new spike and ACE2, given its similarity to the SARS-CoV spike, but we think this may serve as one explanation as to why this virus spreads so much more easily." He says the UT team also noticed the furin cleavage site separating the receptor-binding subunits and the fusion subunits of the spike as soon as the genomic sequence of this novel virus was released, as have several other groups. In the paper $^{3}$, the team notes the spike protein is a fusion protein with a pre-fusion conformation that "undergoes a dramatic structural rearrangement" to fuse with the membrane of the cell the virus is invading. Cryo-EM let the team to resolve different types of 'breathing' motions and conformational changes that might be lost when molecules are locked into a crystal lattice, says Wrapp. "The most dramatic movement that we observed in our dataset is the hinge-like motion of the receptor-binding domain, which is responsible for engaging ACE2 on the surface of cells." Several groups have hypothesized that this motion is an immune evasion strategy: the virus can mask parts that are susceptible to being neutralized, and, in the time gained, it binds to and infects a host cell.

The team determined many of the glycans covering the spike. Glycans are another way viruses prevent antibody binding, says Wrapp. The immune system's antibodies are highly specific for protein motifs, but, for example, a small change can cause a sugar molecule to be attached to a part of the viral protein. This sugar-coating is often called a "glycan shield," he says. The team generated over 3,200 micrograph movies, which led to a $3.5 \AA$-resolution structure of the SARS-CoV-2 spike glycoprotein. Processing these movies is possible now that cryo-EM has become so automated that "our entire data collection only took 24 hours." The UT team regularly performs experiments like these, "but it's hard not to feel a heightened sense of urgency when there is an ongoing pandemic," he says. With some accrued expertise in this field, "I think we all feel obligated to try to contribute to the global efforts that are underway to design vaccines and treatments."

"With crisis comes opportunities for rocket speed progress from highly experienced labs," says Alvin Chew, a PhD student from Singapore and an emerging structural biologist. But COVID-19 intercepted his scientific trajectory. He was on a three-month European Molecular Biology Organization fellowship at Germany's Max Planck Institute of Molecular Physiology. His ambitious plan was, in that time, to learn about cryo-EM software and take his membrane protein target through sample preparation and data collection to get a working model before returning to Singapore. Instead, he had to cut short his stay and hurry back to a 14-day quarantine in Singapore. He sees the structural biology community rising to the challenge and is happy to see informative cryo-EM structures being made public quickly.

\section{PCR and issues}

Having quick data about the virus is enabling genomic research and assay development. But there are challenges. After all, the normal course of affairs is not a pandemic. The University of Birmingham's Loman is a principal investigator in the ARTIC network, which stands for Advancing Real-Time Infection Control, in which collaborators from several universities in the United Kingdom, the United States and Belgium devoted to real-time molecular epidemiology for outbreak response have developed wet-lab and software protocols for characterizing SARS-COV-2 on nanopore sequencers in eight hours. It can be done more quickly if one is doing just a single sample, "but typically people multiplex, so a working day is about right," he says. Josh Quick, Loman's collaborator at the 
University of Birmingham, designed a tiling scheme to amplify the entire viral genome. Soon after the epidemic began, the team developed a multiplexed PCR primer set for whole-genome analysis of the virus. Kentaro Itokawa and colleagues at the National Institute of Infectious Diseases in Tokyo noticed, however, that the method led to low coverage of certain genomic locations ${ }^{5}$. This was because two particular primers could anneal to one another and form dimers, making them less efficient at amplification. The team in Japan developed replacement primers that the ARTIC team adopted. When primers form dimers, primers do not bind efficiently to the template, says Loman. "We are very grateful to Itokawa for finding the solution to this and have updated the scheme," he says. "The speed of open science here is wonderful to behold."

Reliable PCR-based assays are needed around the world and at scale to address what has become a pandemic. The technique is well known, but no individual test was ready for all to use on patient samples. A virus needs no approval to cross borders. But each country has regulations, so PCR needed to begin crossing borders in new ways, especially once the World Health Organization (WHO) declared the outbreak of the coronavirus a Public Health Emergency of International Concern, on January 30.

\section{Cross-border PCR}

Starting in January, the WHO began making molecular assay protocols available from labs willing to share them. The packet includes protocols, primers and probes from the China Center for Disease Control, the Institut Pasteur, the US Centers for Disease Control (CDC) and others.

One of the tests on the WHO site is from an international group of teams at the Charité Institute of Virology, other groups in Germany, and labs in France, the Netherlands, Belgium and China. On January 13, they provided to WHO their workflow and their primer and probe sequences for detecting the virus with RT-PCR. They showed their validation steps and how they measured aspects such as sensitivity and cross-reactivity. They published the workflow ${ }^{6}$ on January 23. The authors describe their accelerated collaboration and how they tweaked the finalized workflow in a week's time. They compared their assay's performance on a wide range of respiratory pathogens in clinical samples. They note that such tasks, along with organizational and logistical issues, would normally take months. It helped to have cross-European networks and collaborations in place from responses

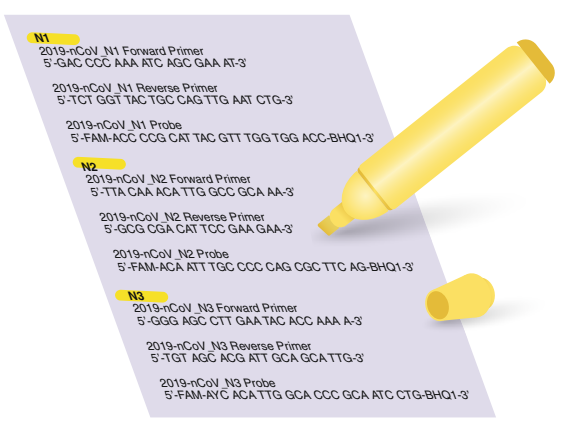

In the US, some public health labs couldn't get the CDC diagnostic kit to work well. Shown here, the original primer-probe sets, named N1, N2, N3. The CDC changed the kit. Credit: E. Dewalt/ Springer Nature

to international health crises in recent years. Overall, they believe the work shows the "enormous response capacity that can be released through coordinated action of academic and public laboratories."

In the United States, before the Secretary of Health and Human Services declared a public health emergency on February 4 , the CDC was the sole US lab allowed to perform clinical diagnostic testing for SARS-CoV-2 with its assay, the CDC 2019-nCoV Real-Time RT-PCR Diagnostic Panel. On that day, FDA granted the CDC an Emergency Use Authorization (EUA) and the test could be sent to qualified labs - those in line with Clinical Laboratory Improvement Amendments of 1988 (CLIA) permitted to perform "high complexity tests."

When Jennifer Rakeman, who heads the New York City Public Health Laboratory, and her colleagues did their initial verification tests of the panel using the CDC's primerprobe sets called N1, N2, N3, she says there were some spurious results with one of the primer-probe sets in the negative controls and negative specimen samples. N1 and N2 are specific to SARS-CoV-2 and N3 is specific to SARS-like viruses, she says. "The positives looked fine, but in some of the negative controls and some of the negative specimens that we needed to test as part of the verification, we saw that one of the primerprobe sets was giving what looked like late amplification," she says. "Initially, there were issues with N3," says Rakeman. "What we found and a small number of other labs found and CDC subsequently found was that there were also some issues, but at a lower frequency, with the N1 primer-probe set." It appeared there was a manufacturing issue with that lot. "It wasn't specific to the way the test was designed, it seemed to be more specific to that particular manufacturing lot of reagents," says Rakeman. Other labs reported issues, too.
According to a spokesperson for Integrated DNA Technologies (IDT), the CDC and FDA contacted the company on February 25 to discuss the CDC EUA testing protocol. That day, IDT shipped primer-probe kits to the CDC. On March 2, the FDA included an IDT-manufactured lot as the first primer and probe kits qualified under the CDC's COVID-19 EUA. The CDC has subsequently qualified additional lots. On March 16, CDC updated its EUA protocol and removed the N3 primers and probes from the kit, and IDT removed $\mathrm{N} 3$ from its kits intended for use with the updated CDC EUA testing protocol. The commercially manufactured primer and probe sets that CDC deems acceptable for this CDC test and that passed testing at CDC are from IDT and Biosearch Technologies. Previously IDT had not manufactured any of the components in the CDC EUA testing protocol.

Separately, Rakeman and her team have purchased IDT primer-probe sets and use them now in an assay developed at Wadsworth Center Laboratory in Albany, New York, for which there is a separate EUA. These primer-probe sets have worked well, she says. The test is identical to the CDC one in terms of forward and reverse primer sequences for N1 and N2. "We use a different extraction method," she says. Initially the CDC required use of an RNA extraction kit from Qiagen, which meant the lab had to purchase the Qiagen instruments, which it didn't have, to do the CDC assay. That Qiagen kit is now in very short supply because so many labs are doing high volumes of testing, says Rakeman. For nucleic acid extraction, the lab uses the bioMérieux EasyMag, an automated platform, "which was something that we were familiar with," she says. The lab has a number of these instruments, "so it's easy for us to pivot to use that platform and those kits." The company is now rationing some of the reagents, she says. Overall, the lab has encountered some supply chain issues, but, she says, "none that have halted testing as of yet."

Hopefully we will learn in time from private labs and state labs whether the CDC diagnostic kit continued to work well for them, despite the initial reagent issue, says Tomer Altman, who consults for small to medium-sized biotech companies on such areas as biochemical pathways and data mining. Because he was curious and wanted to help, he studied the issues with the CDC primers. But rather than finding ways to make his primers better, Altman says he found "the CDC primers had technical flaws in them that went against the conventional wisdom of how to design primers." Primer-probe pairs N1 and N3 
had reverse primers with high-temperature hairpin loops, he says. The N2 primer-probe set had a run of five ' $C$ ' bases in a row on the probe sequence. Primer3, an established software tool, "flagged them as problems," he says. Hairpin loops can lead to inefficient amplification, which theoretically could lead to tests that provide various types of indeterminate results. He does not know if these caused the test's spurious results. But given the technical flaws, in his view, these should never have been put into the primerprobe set sequences for a critical test. He hopes the CDC process will eventually be reviewed by an expert panel, both in terms of the bioinformatics side and wet-lab validation. Read more about this analysis at our blog's News channel.

"I think this makes sense," says Loman, referring to Altman's analysis. "It was disappointing the CDC came out with a pretty poor primer design first off, when there were better ones available from other groups, such as the Hong Kong set," he says, referring to primers developed at the University of Hong Kong. Ghent University researcher Jo Vandesompele has not studied Altman's analysis in detail but says "hairpins may be problematic, but are not always." One limitation of the analysis is that it only mentions software predictions, and wet-lab data would be needed. In wet-lab experiments by postdoctoral fellow Benjamin Nilsson-Payant in tenOever's lab at Mount Sinai, the CDC primers had much background noise, says tenOever.

With clinical testing of a pathogen what matters most, says Vandesompele, is the positive and negative predictive value, so users can be sure about both a positive and a negative test result. Other considerations are cost and turnaround time. Primer sequences matter, as do aspects of sample preparation, nucleic acid purification, reverse transcription, and PCR reaction conditions, such as the concentrations of primers, enzyme, buffer, the temperature cycling protocol and other factors. The reverse transcription step, needed in the case of an RNA virus, is "an often overlooked but critical step" that, among other aspects, determines analytical sensitivity, he says.

\section{PCR, accelerated}

In mid-March FDA issued a guidance to accelerate the availability of assays to be used for COVID-19 under an EUA. A series of approvals began, such as for Cepheid's Xpert Xpress SARS-CoV-2 test, RT-PCR assays run on the company's instruments GeneXpert Instrument System. David Persing, the company's chief medical and technology

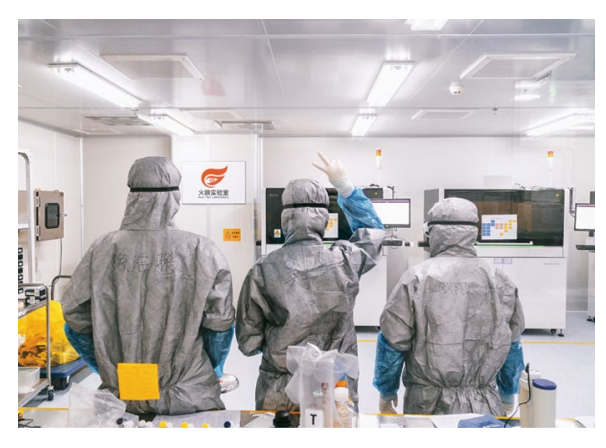

hairpin or dimer structures and adjusted the probes to avoid such issues, says Chen. As of early April, the company has manufactured and distributed 4.72 million tests to 70 countries and has completed 520,000 tests in its own labs. The company says it has ramped up to manufacture two million tests a day. BGI designed the Huo-Yan or, 'Fire Eye,' Lab in Wuhan. It was built, he says, in five days and can do 10,000 tests a day.

Among some general worries, says These technicians are in a BGI-designed lab in Wuhan, China with instruments from several companies. According to BGI it was built in five days and can do 10,000 tests a day. Credit: BGI

officer, says in a video that results are generated in $45 \mathrm{~min}$ or less. There is Roche Molecular Diagnostics' cobas SARS-CoV-2 test, an RT-PCR system for use with dedicated instruments. There is Thermo Fisher's TaqPath COVID Combo Kit, to be used with the Applied Biosystems RT-PCR instrument. A Thermo Fisher spokesperson tells Nature Methods the plan is to ramp up production to five million tests a week.

RT-PCR tests are suitable for early screening, and sequencing-based kits are used for concurrent detection of infection or to confirm results in complicated infections, says Weijun Chen, chief scientist of infectious disease at BGI Research, whose comments were relayed through a spokesperson. "The same types of kits have different potency," says Chen. More sensitive kits should be selected first, but the cost may be higher. In late January, BGI received emergency approval for its RT-PCR kit from China's National Medical Products Administration, and in late March it received an EUA from the FDA. Tests need to have been tested in the "real world," he says. They need to generate results quickly and be amenable to scaling for population-wide screening, and "you want to use what you already have in the lab to start the test right away, not to wait to buy a new machine." The BGI team ran cross-reactivity assays against, for example, common respiratory viruses and bacterial cultures, and tested their assay on samples from patients with confirmed COVID-19 disease. To find the minimum detection threshold, the team used serial dilutions of clinically positive samples with known concentrations and repeated the experiments at least 20 times. They evaluated the shelf life of the test reagents and overall test kit stability. For primer design, they selected specific genomic regions of the virus, designed primers and probes in silico, checked them for
Loman, is a shortage of lysis buffer and reverse transcriptase enzymes from certain suppliers, which risks putting a crimp in testing. Vandesompele echoes the concern. $\mathrm{He}$ is also chief scientific officer and cofounder of the biotech Biogazelle, which the Belgian government has tasked, along with Johnson \& Johnson, GlaxoSmithKline and Thermo Fisher in collaboration with clinical labs and universities, with increasing PCR testing capacity. Given high demand, companies cannot always deliver supplies quickly. With backordering can come shortages of, for example, lysis reagents, binding reagents, washing reagents and elution reagents. You can read more about emerging assays at our our blog's News channel.

Academic and industry labs are hurrying to address the COVID-19 pandemic. At Zymo Research, applications specialist Luigi Basilio says he and his colleagues see the company culture rising to the challenge of a pandemic. "RNA extraction is certainly something we never thought we'd see trending in global news or on social media, but I think it's created a learning opportunity for people who aren't in the research space," he says. Perhaps this shows why RNA extraction, among other tools and techniques, is so important. "As critical as RNA extraction is, so are the scientists and researchers who perform them," he says. "Who knows, maybe all of this will inspire young people to take interest and create a new generation of scientists."

\section{Vivien Marx ${ }^{凶}$ \\ Technology editor for Nature Methods.

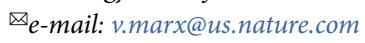

Published online: 17 April 2020

https://doi.org/10.1038/s41592-020-0827-7

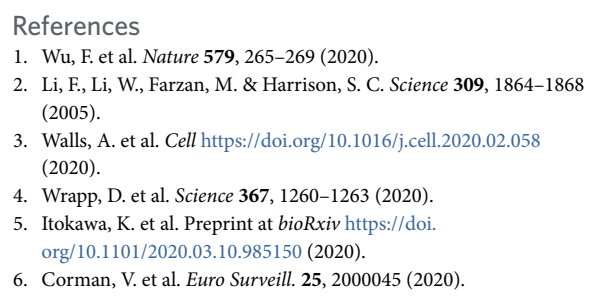

